\section{Serotonin Syndrome: A Real Risk of Anti-migraine Therapy}

A 13-year-old girl, diagnosed as migraine without aura was under our follow-up for last one year. She was doing well on flunarizine and rarely required nonsteroidal anti-inflammatory drugs (NSAIDs) or rizatriptan for acute attacks. However, following the poor academic performance in the terminal examination, she developed depressive features including poor sleep, anorexia, decreased interest in the study, withdrawal from leisure activities and other daily routine work, decreased interaction with parents, emotional lability and inconsolable crying even on the minimal conversation regarding resumption of academic activities. She also developed an increased frequency of acute attacks of migraine, especially after prolonged periods of insomnia and hunger. Given the above features, she was prescribed amitriptyline $25 \mathrm{mg}$ /day by a local practitioner. However, after consuming amitriptyline and rizatriptan daily for five days, she developed worsening of symptoms along with excessive anxiety, agitation, dizziness, palpitation, sweating and tremors of hands. She was then referred to our center with a diagnosis of conversion disorder.

On evaluation, she was found to have temperature of $38.7^{\circ} \mathrm{C}$, diarrhea, vomiting and generalized hyperreflexia. Hematological and biochemical parameters, thyroid profile and MRI brain studies were within normal limits. Urine drug screen was negative for metabolites of any addictive drug. Serotonin syndrome scale score was 11 , suggestive of serotonin toxicity. Both rizatriptan and amitriptyline were discontinued and she was started on oral lorazepam for anxiety and agitation. Within 48 hours, the symptoms dramatically improved and the serotonin syndrome scale score reduced to 2 . She was advised flunarizine and naproxen for migraine and low dose olanzapine for depressive symptoms, apart from cognitive behavioral therapy.
Serotonin syndrome, caused by increased serotonin levels in the body, may occur as an adverse drug reaction after using some serotonergic medications in combination or overdose of such medications [1,2]. While rizatriptan is a 5-HT $\mathrm{H}_{1 \mathrm{~B} / \mathrm{D}}$ receptor agonist, amitriptyline is a tricyclic antidepressant. Both the drugs increase serotonergic activity in the body by inhibition of the uptake of serotonin and norepinephrine in adrenergic and serotonergic neurons by acting on the membrane pump mechanism [3]. An alert was issued by Food and Drug Administration (FDA) in 2006 on the possibility of lifethreatening serotonin syndrome when triptans are combined with selective serotonin receptor inhibitors (SSRIs) and selective norepinephrine receptor inhibitors (SNRIs) [4]. Although this combination is less commonly prescribed in children, pediatricians still need to be aware of this entity for timely diagnosis and prompt treatment.

Inder Kumar Sharawat and Prateek Kumar Panda* Pediatric Neurology Unit, Department of Pediatrics, All India Institute of Medical Sciences, Rishikesh, Uttarakhand, India. *drprateekpanda@gmail.com

\section{REFERENCES}

1. Scotton WJ, Hill LJ, Williams AC, Barnes NM. Serotonin syndrome: Pathophysiology, clinical features, management, and potential future directions. Int $\mathbf{J}$ Tryptophan Res. 2019;12:1178646919873925.

2. Park SH, Wackernah RC, Stimmel GL. Serotonin syndrome: Is it a reason to avoid the use of tramadol with antidepressants? J Pharm Pract. 2014;27:71-8.

3. Hegerl U, Bottlender R, Gallinat J, Kuss HJ, Ackenheil M, Möller HJ. The serotonin syndrome scale: first results on validity. Eur Arch Psychiatry Clin Neurosci. 1998;248: 96-103.

4. Evans RW. The FDA alert on serotonin syndrome with combined use of SSRIs or SNRIs and triptans: An analysis of the 29 case reports. Med Gen Med. 2007;9:48. 